\title{
Electrophoretic deposition of Au NPs on CNT networks for sensitive $\mathrm{NO}_{2}$ detection
}

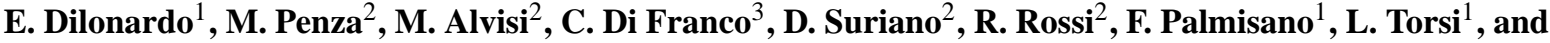 \\ N. Cioffi ${ }^{1}$ \\ ${ }^{1}$ Department of Chemistry, Università degli Studi di Bari Aldo Moro, Bari, Italy \\ ${ }^{2}$ ENEA, Italian National Agency for New Technologies, Energy and Sustainable Economic Development, \\ Technical Unit for Materials Technologies - Brindisi Research Center, Brindisi, Italy \\ ${ }^{3}$ CNR-IFN Bari, Bari, Italy
}

Correspondence to: E. Dilonardo (elena.dilonardo@uniba.it) and N. Cioffi (nicola.cioffi@uniba.it).

Received: 31 July 2014 - Revised: 24 September 2014 - Accepted: 27 September 2014 - Published: 16 October 2014

\begin{abstract}
In the present study, Au-surfactant core-shell colloidal nanoparticles (NPs) with controlled dimension and composition were synthesized by sacrificial anode electrolysis. Transmission electron microscopy (TEM) revealed that Au NPs core diameter is between 8 and $12 \mathrm{~nm}$, as a function of the electrosynthesis conditions. Moreover, surface spectroscopic characterization by X-ray photoelectron spectroscopy (XPS) analysis confirmed the presence of nanosized gold phase. Controlled amounts of Au NPs were then deposited electrophoretically on carbon nanotube (CNT) networked films. The resulting hybrid materials were morphologically and chemically characterized using TEM, SEM (scanning electron microscopy) and XPS analyses, which revealed the presence of nanoscale gold, and its successful deposition on CNTs. Au NP/CNT networked films were tested as active layers in a two-pole resistive $\mathrm{NO}_{2}$ sensor for sub-ppm detection in the temperature range of $100-200^{\circ} \mathrm{C}$. $\mathrm{Au}$ $\mathrm{NP} / \mathrm{CNT}$ exhibited a p-type response with a decrease in the electrical resistance upon exposure to oxidizing $\mathrm{NO}_{2}$ gas and an increase in resistance upon exposure to reducing gases (e.g. $\mathrm{NH}_{3}$ ). It was also demonstrated that the sensitivity of the Au NP/CNT-based sensors depends on Au loading; therefore, the impact of the Au loading on gas sensing performance was investigated as a function of the working temperature, gas concentration and interfering gases.
\end{abstract}

\section{Introduction}

In recent years the use of carbon nanotubes (CNTs) has attracted great interest in gas sensing applications because of their reduced dimensionality, which means a high surfaceto-volume ratio, together with an outstanding gas adsorption capability, and lower operating temperatures compared to the conventional metal oxide-based device; therefore, these properties make CNTs ideal candidates for environmental sensing applications. Presently, continued progress in CNT-based sensor development for gas detection has been achieved (Zhang et al., 2008; Bondavalli et al., 2009; Penza et al., 2014).

Semiconducting CNTs generally have a typical p-type electrical behaviour under specific ambient conditions (Martel et al., 1998): in the presence of adsorbing oxidizing gases, their resistance decreases; instead, the resistance variation is in the opposite direction in the presence of reducing gas molecules (Kong, 2000). The possible sensing mechanisms include electrostatic gating (Kong, 2000), interaction with pre-adsorbed oxygen species (Collins et al., 2000), charge transfer from adsorbed gas species to carbon nanotubes (Chang et al., 2001; Zhao et al., 2002), and alteration of the electrode work function which leads to a change in the carrier mobility due to formation or removal of the Schottky barrier (Peng et al., 2009).

Although significant progress has been made in understanding the sensing mechanisms of pristine CNTs towards gas molecules, the operation/sensing mode still remains ambiguous (Fan et al., 2005). 
The need for air-quality monitoring necessitates the development of highly sensitive sensors that are selective for the detection of individual pollutant gases, especially $\mathrm{NO}_{2}$ which is a very toxic air pollutant to be detected at sub-ppm level with high sensitivity and selectivity.

The sensing response of gas sensors based on unmodified CNTs is weak and scarcely selective since the ideal carbon hexagonal network is held together by strong $\mathrm{sp}^{2}$ bonds characterized by a low chemical reactivity with the molecular environment (Peng and Cho, 2003). Consequently, the functionalization of the CNT sidewalls is mandatory to improve both the sensitivity and the selectivity of the CNT-based gas sensors.

In general, molecular sensing requires strong interactions between sensor material and target molecules; this is also the case for nanotubes. It has been found that nanotubes are scarcely sensitive to many types of molecules (e.g. hydrogen and carbon monoxide), indicating an apparent lack of specific interactions between nanotubes and these molecules; therefore, nanotube sensors with molecular selectivity can be obtained through rational chemical and/or physical modification of the nanotube's surface, involving simple deposition of functional materials on the nanotubes (Kong et al., 2001).

Moreover, it has been reported that the sensitivity of nanotube gas sensors could be enhanced through functionalization and defect generation, because single-walled carbon nanotube (SWNT) defect sites of various nature, such as topological, rehybridization, incomplete bonding defects and doping with elements other than carbon (Charlier, 2002), are more reactive than the pristine $\mathrm{sp}^{2}$-bonded lattice. Appropriate deposition procedures can selectively decorate CNT defect sites with catalytic nanophases (Fan et al., 2005). As reported in Robinson et al. (2006), CNT defect sites play an important role in the electrical response for a broad spectrum of chemical vapours, and the controlled introduction of defects can be used to increase the sensitivity and chemical selectivity of both the conductance and capacitance responses. The defects form low-energy adsorption sites that also serve as nucleation sites for analyte condensation at high vapour concentrations; moreover, the chemical sensitivity of SWNTs can be increased significantly by introducing a controlled low density of defects along the nanotube sidewall. In addition, experimental studies (Lv et al., 2010) showed that defects of CNTs created nucleation sites for metal nanoparticles. In fact, the structural defects, such as topological defects and vacancies in CNTs, always existed in most of the CNTs. Studies (Kim et al., 2007; Pannopard et al., 2009) have indicated that the transition metal has a rich d-electron and empty orbit, and the small gas molecule can bond strongly to the metal when adsorbed on the surface. As previously reported (Kim et al., 2007; Zhang et al., 2014), when metal nanoparticles are deposited on the perfect surface of a nanotube or away from the surface defects, the interaction between the metal and the nanotube is weak; on the other hand, when the metal is adsorbed on the point defect site of the nanotube surface, the structure becomes stable.

Covalent and non-covalent methods have been employed to functionalize CNTs with various materials including polymers (Salavagione et al., 2014), metal oxides (Zhang et al., 2013), metals (Penza et al., 2014) and organometallic complex (Brunet et al., 2012). In particular, the functionalization with metal nanoparticles (NPs) can lead to highly sensitive and selective gas sensors thanks to the extraordinary catalytic properties of the metal NPs (Feldheim and Foss, 2002), as already suggested by several experimental (Khalap et al., 2010), theoretical (Pannopard et al., 2009) and combined (Kauffman et al., 2010) works.

Leghrib et al. (2010) reported on multi-walled carbon nanotubes (MWCNTs) decorated with different metal NPs, e.g. $\mathrm{Rh}, \mathrm{Pd}, \mathrm{Au}$ and $\mathrm{Ni}$, to tailor gas recognition of benzene vapours at concentrations lower than $50 \mathrm{ppb}$ with high sensitivity and selectivity at room temperature. Au- or Agdecorated CNT films have been used to detect $\mathrm{NO}_{2}$ at up to $500 \mathrm{ppb}$ at room temperature (Espinosa et al., 2007). Star et al. (2006) and also Lu et al. (2004) decorated single-walled CNTs with $\mathrm{Pt}, \mathrm{Pd}, \mathrm{Au}$ or $\mathrm{Rh}$ for detecting a large variety of gases such as $\mathrm{CO}, \mathrm{NO}_{2}, \mathrm{CH}_{4}, \mathrm{H}_{2} \mathrm{~S}, \mathrm{NH}_{3}$ and $\mathrm{H}_{2}$, by discriminating from interfering gases. Moreover, recent works (Colindres et al., 2014; Doroodmand et al., 2013) concern the functionalization of CNTs with $\mathrm{Pd}$ and the electrodeposition of $\mathrm{FeOOH}$ nanostructures on $\mathrm{CNT}$ s for $\mathrm{O}_{3}$ gas sensing, with a detection limit in the range of ppb and a high selectivity.

Among various metal NPs, Au-functionalized CNT-based resistive gas sensors show the best sensitivity and selectivity towards $\mathrm{NO}_{2}$ gas detection (Penza et al., 2007).

Several methods have been used to functionalize CNTs by metal NPs, such as thermal evaporation (Scarselli et al., 2012), sputtering (Penza et al., 2011) and electrochemical deposition (Mubeen et al., 2011).

In this study, we report on the functionalization of the CNT-based gas sensor by Au NPs with defined dimension and controlled loading using an electrophoretic deposition, as an easier process for the mass production of metalfunctionalized CNT-based gas sensor devices. The effects of the operating sensor temperature (range $100-200^{\circ} \mathrm{C}$ ) and of the metal loading on sensor performance towards $\mathrm{NO}_{2}$ detection were reported. Moreover, the Au NP-functionalized CNT-based gas sensor response in the presence of interfering gases (e.g. $\mathrm{NO}, \mathrm{NH}_{3}$ ) was also evaluated.

\section{Experimental}

\subsection{Electrochemical synthesis of Au NPs}

The core-shell gold nanoparticle (Au NP) solution, used to metal directly decorate CNT-based gas sensors, was produced by the sacrificial anode electrolysis (SAE), in the presence of $0.05 \mathrm{M}$ tetraoctylammonium chloride (TOAC), with the simultaneous function of electrolyte and Au NP 


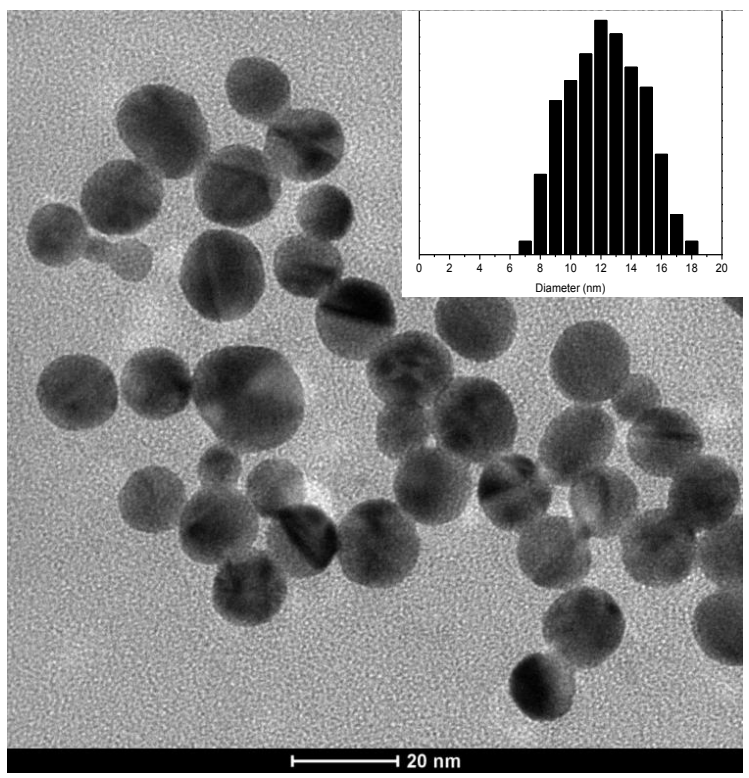

Figure 1. TEM image and dimensional dispersion histogram (inset) of electrochemically synthesized core-shell Au NPs/TOAC.

stabilizer, dissolved in an anhydrous tetrahydrofuran (THF) and acetonitrile $(\mathrm{ACN})$ solution, mixed in $3: 1$ ratio (Cioffi et al., 2011). A three-electrode cell was used, equipped with an $\mathrm{Ag} / \mathrm{AgNO}_{3}(0.1 \mathrm{M}$ in $\mathrm{ACN})$ reference electrode, a gold anode and a platinum cathode. During the process, the cell was kept under a nitrogen atmosphere.

Since in these systems the NPs shell thickness is roughly proportional to the chain length of the surfactant (Reetz and Helbig, 1994; Reetz et al., 1995), while the core size is mainly influenced by the electrochemical parameters, $\mathrm{Au}$ NPs with a shell thickness of about $1.2 \mathrm{~nm}$ were synthesized using TOAC. The working potential was set at $+1 \mathrm{~V}$ corrosion voltages and the electrolysis charge at $300 \mathrm{mC}$. As discussed in details in previous works (Cioffi et al., 2011), and reported in Fig. 1, the electrochemically synthesized Au NPs have a uniform dispersion with diameter of $12 \mathrm{~nm}$. Moreover, since the Au NPs are stabilized by the tetraoctylammonium surfactant with a positive charge, as revealed in previous work of our group (Cioffi et al., 2000; Ieva et al., 2008), the net surface charge of metal NPs is positive and it was used for the electrophoretic deposition to surface decorate CNT devices.

\subsection{Growth of carbon nanotubes and sensor fabrication}

MWCNT layers were prepared by a radio-frequency plasmaenhanced chemical vapour deposition (RF-PECVD) system, at a reasonably low growth temperature of $450{ }^{\circ} \mathrm{C}$, onto low-cost alumina substrates $(10 \mathrm{~mm}$ width $\times 10 \mathrm{~mm}$ length $\times 0.6 \mathrm{~mm}$ thickness). The experimental conditions used, as well as details about MWCNT composition,

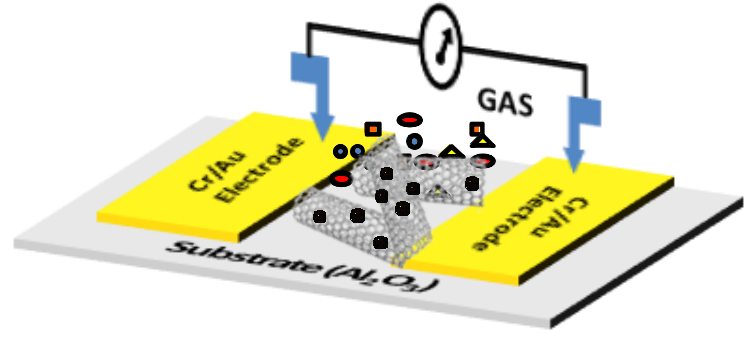

Figure 2. Scheme of Au-modified CNT-based gas sensor device.

structure, and abundance of amorphous matter, are reported elsewhere (Penza et al., 2008).

Modification of CNT-based devices was performed using an electrophoretic process consisting of a three-cell electrode in which the anode was a Pt foil, the cathode was the CNT device and $\mathrm{Ag} / \mathrm{AgNO}_{3}$ was the reference electrode. The distance between the anode and the cathode was set at $2 \mathrm{~cm}$. The electrolytic solution was the Au NP colloidal solution. The deposition process is a cathodic process in which an applied working potential a little bit more negative than the open circuit potential was used to induce the migration of the colloidal Au NPs towards the negative cathode (CNT device), the reduction of the residual gold ions in the positive shell followed by its removal from the metal NPs surface, with the final result of the CNT surface decorated with Au NPs. The excess of the surfactant from the functionalized device was completely removed by washing it with $\mathrm{ACN}$ for three times.

The process was performed using two different deposition times, 90 and $600 \mathrm{~s}$, to deposit different metal loadings. The scheme of a $\mathrm{Au} / \mathrm{CNT}$ resistive gas sensor device is reported in Fig. 2.

\subsection{Material characterization set-up}

Surface chemical characterization was performed by means of a Thermo VG Theta Probe XPS spectrometer equipped with a $\mu$ spot monochromatic $\mathrm{Al} \mathrm{K \alpha}$ source. Both survey and high-resolution spectra were acquired in fixed analyser transmission mode with pass energies of 150 and $100 \mathrm{eV}$, respectively.

The dimension of synthesized Au NPs was evaluated using a FEI TECNAI T12 transmission electron microscopy (TEM) instrument.

The morphology of pristine and Au-decorated CNTs was analysed by scanning electron microscopy (SEM), using a Field Emission Zeiss $\Sigma$ IGMA instrument at 5-10 KV, $10 \mu \mathrm{m}$ aperture, directly on the sensor devices.

\subsection{Gas sensing measurements}

The experimental set-up used for gas sensing measurements is reported elsewhere (Penza et al., 2010). The CNT-based gas sensors were placed in a sealed stainless test cell $(500 \mathrm{~mL}$ 
Table 1. XPS surface chemical composition of pristine and Aufunctionalized CNTs.

\begin{tabular}{llll}
\hline Sample & $\mathrm{C}(\%)$ & $\mathrm{Au}(\%)$ & $\mathrm{O}(\%)$ \\
\hline Pristine CNTs & $95.0 \pm 0.5$ & - & $5.0 \pm 0.5$ \\
$\mathrm{Au}$ NPs/CNTs $t: 90 \mathrm{~s}$ & $94.4 \pm 0.5$ & $0.3 \pm 0.2$ & $5.3 \pm 0.5$ \\
$\mathrm{Au}$ NPs/CNTs $t: 600 \mathrm{~s}$ & $91.2 \pm 0.5$ & $1.1 \pm 0.2$ & $7.8 \pm 0.5$ \\
\hline
\end{tabular}

volume) for gas exposure measurements. They were in thermal contact with a home-made heater sink, powered by a DC power supply system (Agilent 6644A, 0-60 V/0-3.5 A), to control the desired set-point operating temperature. The DC electrical conductance of the metal oxide-based gas sensors was measured by the volt-amperometric technique in the two-pole format by a multi-metre (Agilent, 34401A). The chemiresistors in an array of four sensing elements were scanned with automated control by a multiplexing switch system (Keithley, 7001) equipped by a low-current scanner card (Keithley, 7158). All data were acquired and stored for further analysis in a PC-based workstation, equipped with software compiled in Agilent-VEE.

Dry air was used as the reference gas and diluting gas to air condition the sensors. The gas flow rate was controlled by distinct mass flow metres (MFC) with different full scales and controlled by GMIX software. The total flow rate per exposure was kept constant at $1000 \mathrm{sccm}$. The gas sensing experiments were performed by measuring the resistance change of deposited films under the exposure to the target gas at different concentrations. The sensing experiments were conducted at three different sensor temperatures (100, 150 and $200^{\circ} \mathrm{C}$ ), to evaluate the temperature effect on gas sensing performance.

The gas sensing cycle consisted of a period (at least $60 \mathrm{~min}$ ) of stabilization of the sensor signals with dry air (analysis certificate on the gas bottle given by the company providing gases, AirLiquide) flowing, an exposure time of $10 \mathrm{~min}$ to various targeted gas concentrations at increasing steps and finally a recovery time (at least $60 \mathrm{~min}$ ) to restore the sensor signals with dry air flowing to clean the test cell and sensor surface.

The sensor response to a given gas concentration was defined as the resistance change, $\Delta R$, where $\Delta R$ is the change in resistance between the values of steady state of the electrical resistance, $R_{f}$ and $R_{i}$, of the sensor upon a target gas and in air, respectively. The mean gas sensitivity $\left(\% \mathrm{ppm}^{-1}\right)$ $\left(S_{\mathrm{m}}\right)$ is defined as weighted mean of the ratio between percentage relative resistance change (\%) over gas concentration unit (ppm); it can be calculated with Eq. (1):

$S_{\mathrm{m}}=\frac{1}{n} \sum_{j=1}^{n} \frac{\left|\frac{\Delta R}{R_{i}}\right|_{j}}{c_{j}}\left(\% \mathrm{ppm}^{-1}\right)$

where $c_{j}$ is a defined gas concentration which corresponds to the $\left[\Delta R / R_{i}\right]_{j}$ response.

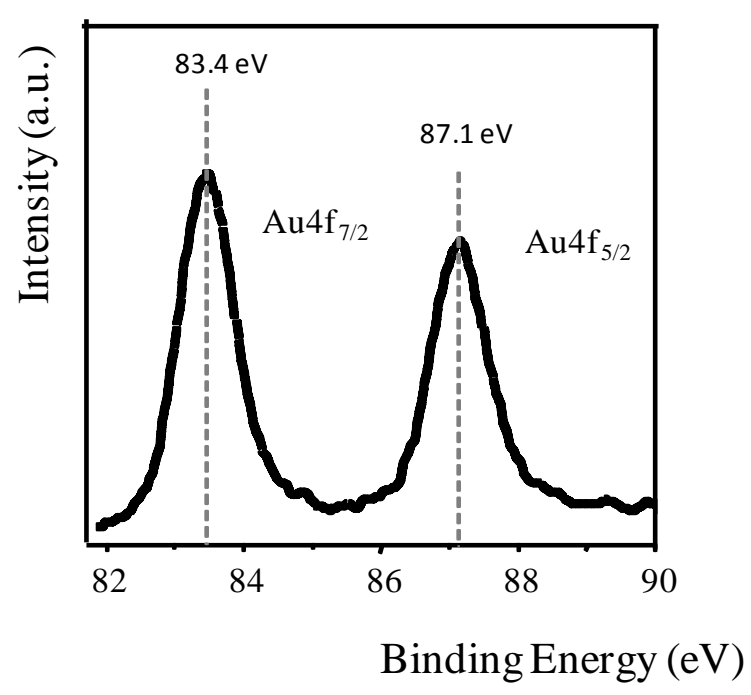

Figure 3. XPS Au4f spectra of Au-decorated CNTs.

\section{Results}

\subsection{Material characterization}

The surface of pristine CNTs and Au-decorated CNTs at different process times was chemically characterized by XPS. The detailed chemical quantification of the structures showed that by increasing the deposition time, as expected, the gold loading on CNTs increases, as reported in Table 1. Therefore, by controlling the process time it is possible to tune the deposited metal concentration.

In Fig. 3 the XPS Au4f spectrum of electrophoretically Au-decorated CNTs is reported. It is composed by a single doublet, attributed to $\mathrm{Au}$ in the elemental oxidation state. The position of the $A u 4 f_{7 / 2}$ peak at $83.7 \mathrm{eV} \pm 0.2 \mathrm{eV}$ was significantly lower than that expected for bulk metallic Au at $84.0 \mathrm{eV}$ (Moulder et al., 1992). This phenomenon is reasonably due to initial state size effects, highlighted for small gold particles (Radnik et al., 2002; Cioffi et at., 2011). Therefore, the effectiveness functionalization of CNTs with nano-size gold particles has been demonstrated. To corroborate these results, morphological analysis was performed by SEM.

Figure 4 displays SEM images of un-functionalized and surface-modified CNT films with of 0.3 and 1.1 at. \% of Au loading. The morphological analysis of CNTs reveals a pronounced tubular structure, consisting of tangled nets of densely distributed chains.

Past Raman spectroscopy on pristine CNTs revealed the presence of some amount of amorphous carbon in the CNTs network (Penza et al., 2008, 2010); specifically, the high intensity ratio $(I(D) / I(G))$ of $D$ peak, associated with the disordered graphitic layer, to $G$ peak, due to the tangential modes of the graphitic planes, revealed qualitatively the presence of a large amount of disorder and defects. On the surface-functionalized CNTs, Au NPs effectively 

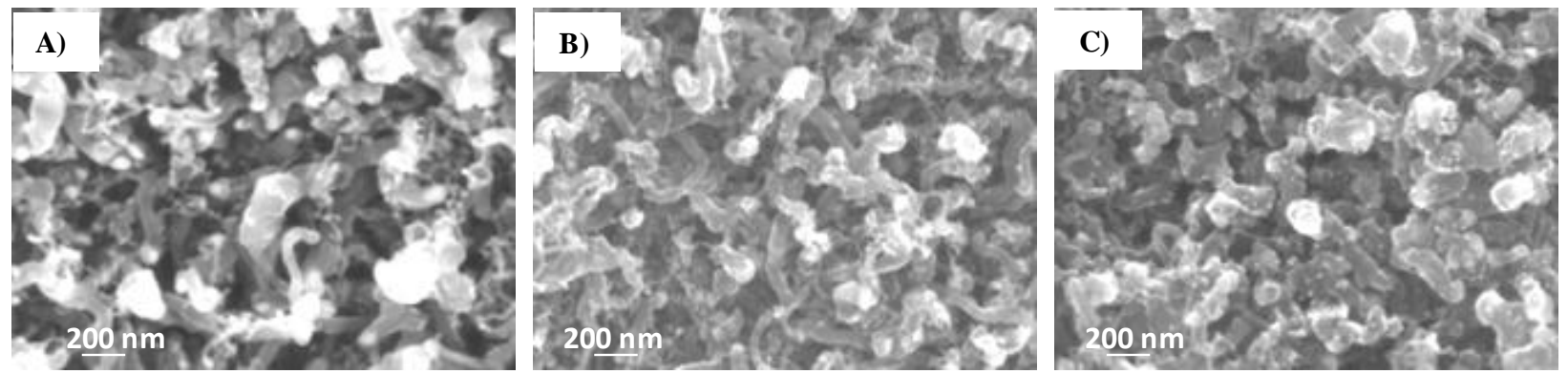

Figure 4. SEM images of (a) pristine CNTs, and CNTs with (b) 0.3 at. $\%$ and (c) 1.1 at. \% Au loading.

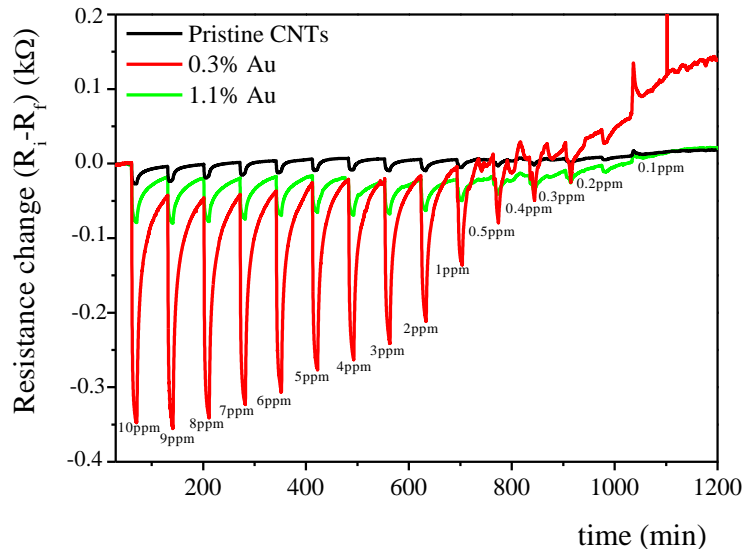

Figure 5. Time response of the $\mathrm{NO}_{2}$ gas chemiresistor based on pristine CNT films, and functionalized with Au-loading of 0.3 and 1.1 at. \%, at a working temperature of $150^{\circ} \mathrm{C}$.

decorate MWCNT sidewalls, forming isolated nanoclusters, with a density increase upon increasing deposited Au content. Specifically, a homogeneous distribution of Au NPs is found on CNT sidewalls in the top layers of films; even the presence of Au NPs on CNT sidewalls in the inner layers should not be excluded due to the film porosity. The tuned surface modification enables CNTs with controlled surface metal loading for the fabrication of selective gas sensors. The catalytic covering of nanotubes should strongly affect their properties concerning the gas adsorption and reactivity, hence, the tailoring of gas sensitivity, as discussed in the next paragraph.

\subsection{Gas sensing characterization}

Figure 5 reports the time response of the CNT chemiresistors, pristine and surface modified with a different Au NPs loading of 0.3 and 1.1 at. \%, exposed to $10 \mathrm{~min}$ pulses of decreasing spot concentrations of $\mathrm{NO}_{2}$ gas in the low range from 10 to $0.1 \mathrm{ppm}$. The working sensor temperature was $150^{\circ} \mathrm{C}$. The electrical resistance of Au-loaded and pristine CNT devices decreases rapidly when exposed to $\mathrm{NO}_{2}$ gas; thus the p-type characteristic is maintained also after functionalization of the
CNTs with Au nanoclusters. In particular, the $\mathrm{NO}_{2}$ sensing response for all sensors increases upon increasing the analyte gas concentration, as reported in the calibration curves in Fig. 6a for all gas sensors. As reported in Fig. 6b in which the $\mathrm{NO}_{2}$ concentration range is magnified from 0 up to $1.0 \mathrm{ppm}$, the trend of the sensor response to $\mathrm{NO}_{2}$ concentration is quite linear up to $1 \mathrm{ppm}$ of $\mathrm{NO}_{2}$; instead, after this value, at higher gas concentrations, the response variation is lower, probably due to the sensor saturation. Moreover, the $\mathrm{NO}_{2}$ response at the operating sensor temperature of $150{ }^{\circ} \mathrm{C}$ is higher for $\mathrm{Au}$ modified CNTs compared to the pristine CNT film; in particular, the CNT sensor containing 0.3 at. \% of Au shows the better response.

The baseline resistance is not stable for all measured functionalized CNT-based gas sensors, due to the thermal drift induced by operating sensor temperature in CNT material, and probably also to the instability of the catalyst particles onto CNT sidewalls, activated by thermal energy (Penza et al., 2008); moreover, the baseline drift is more evident at low gas concentration because of the lower ratio between signal and noise.

Considering the sensor operating temperature effect on sensing properties of all gas sensor devices at all investigated temperatures in the range $100-200^{\circ} \mathrm{C}$, the mean sensitivity towards $\mathrm{NO}_{2}$ of Au-doped CNT films is always higher than the un-doped, as reported in Fig. 7; specifically, the highest values are obtained for CNTs containing the lower Au loading of 0.3 at. \% , especially at lowest tested temperatures. Moreover, for all gas sensor devices the maximum of mean sensitivity is obtained at the intermediate temperature of $150^{\circ} \mathrm{C}$ at which both systems, Au NPs and CNTs, are stable, since at higher temperature CNTs could decompose and Au NPs could agglomerate, decreasing their catalytic properties. On the contrary, a lower temperature could not be enough to activate chemically effective interactions at the interfaces between the two structures, probably responsible of the dominant sensing mechanisms. The higher sensor response towards $\mathrm{NO}_{2}$ of CNTs containing the lower loading of Au NPs is probably due to the fact that in this condition the Au NPs agglomeration in nanoclusters of higher dimension is prevented, since the surface density of Au NPs 

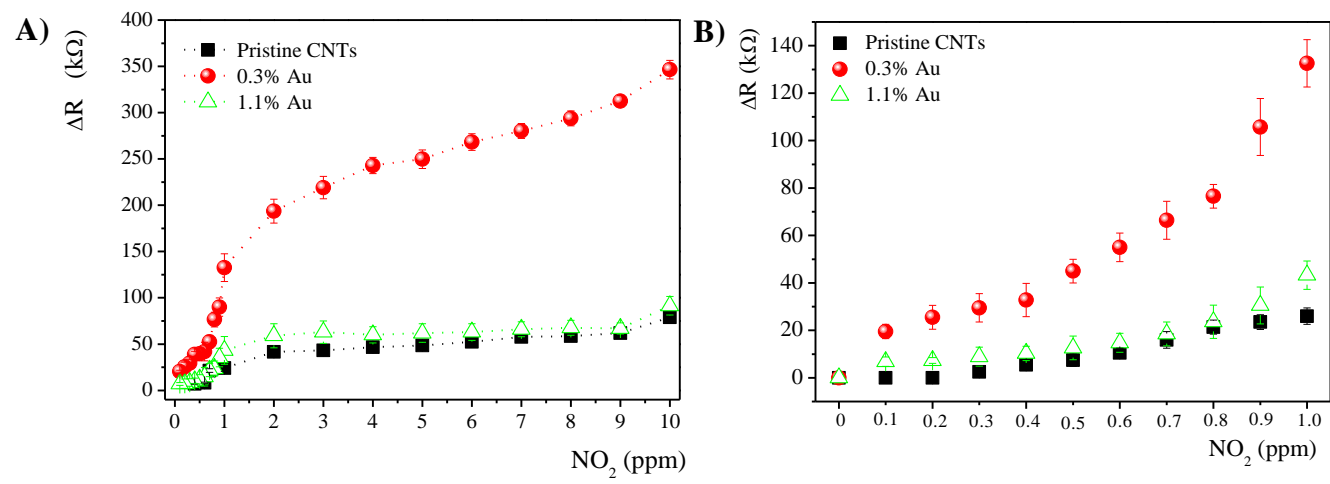

Figure 6. Calibration curves for pristine and Au-decorated CNT-based sensors toward $\mathrm{NO}_{2}$ gas at $150{ }^{\circ} \mathrm{C}(\mathbf{a})$ in the concentration range of $0.1-10 \mathrm{ppm}$ and (b) the magnification of $0.1-1.0 \mathrm{ppm}$ concentration range.

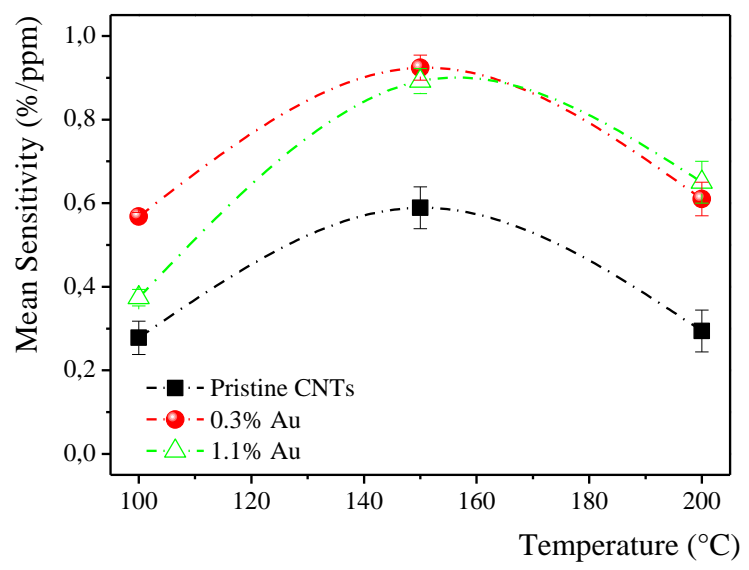

Figure 7. Mean sensitivity of pristine and Au-decorated CNT-based sensors toward $\mathrm{NO}_{2}$ gas at different sensor operating temperatures in the range $100-200^{\circ} \mathrm{C}$.

on the sidewall of CNT is low, as revealed by SEM images; therefore, a high surface catalytic activity of nanosized Au is guaranteed. Instead, a high Au loading causes a high surface density of the Au NPs that could agglomerate, reducing their catalytic properties. As confirming this assumption, Hvolbaek et al. (2007) have demonstrated by density functional calculation that the fraction of low coordinated Au atoms scales approximately with the catalytic activity, suggesting that atoms on the corners and edges of the Au NPs are the active sites.

Moreover, excellent short-term repeatability of the response to the selected targeted gases has been also measured for the Au-modified CNT-based sensors, as reported in Fig. 8 in which the repeatability test has been evaluated exposing functionalized CNTs with Au-loading of 0.3 at. \% at 10 and $1 \mathrm{ppm}$ of $\mathrm{NO}_{2}$ repeating 10 times the exposure to each gas concentration, at a working temperature of $150{ }^{\circ} \mathrm{C}$.

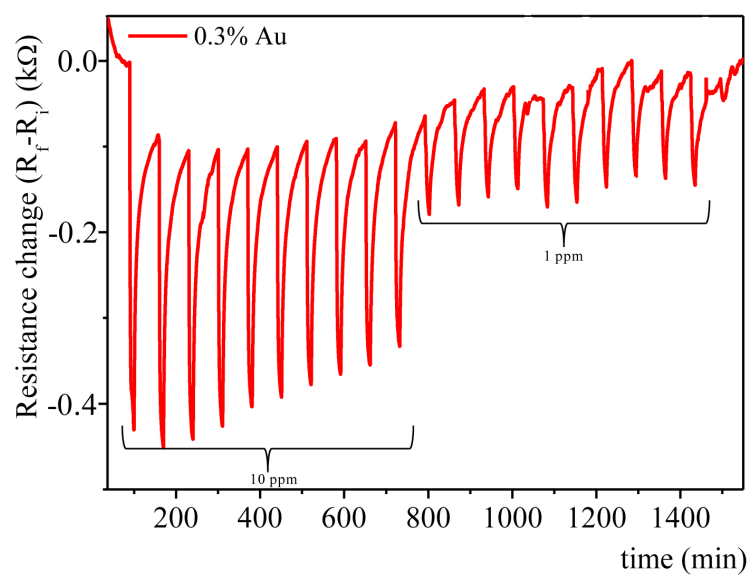

Figure 8. Repeatability test based on time response of functionalized CNTs with Au loading of 0.3 at. $\%$ at a working temperature of $150^{\circ} \mathrm{C}$ and at 10 and $1 \mathrm{ppm}$ of $\mathrm{NO}_{2}$, repeating 10 times the exposure to each gas concentration.

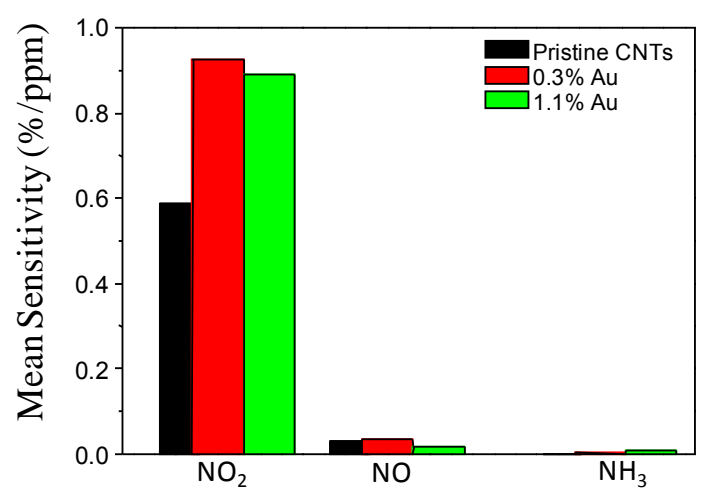

Figure 9. Mean sensitivity of CNT-based sensors towards $\mathrm{NO}_{2}, \mathrm{NO}$ and $\mathrm{NH}_{3}$ gases at a sensor temperature of $150^{\circ} \mathrm{C}$. 
The cross-sensitivity of CNT sensors, un-modified and $\mathrm{Au}$ loaded, has been measured towards $\mathrm{NH}_{3}$ and $\mathrm{NO}$ gases at a sensor operating temperature of $150^{\circ} \mathrm{C}$ and, as reported in Fig. 9, all CNT-based devices are selective towards $\mathrm{NO}_{2}$ detection with a higher sensitivity at about 1 order of magnitude. Finally, Au-modified CNT gas sensors exhibit a negligible response towards $\mathrm{CO}, \mathrm{CH}_{4}$ and $\mathrm{SO}_{2}$ gases, as measured repeatedly (not shown).

\section{Conclusions}

In conclusion, CNT-based sensor devices have been directly decorated with Au NPs by means of an electrophoretic deposition method, for $\mathrm{NO}_{2}$ gas detection at operating temperature in the range of $100-200^{\circ} \mathrm{C}$. The surface modification of the CNT networked films with size- and loading-controlled $\mathrm{Au}$ nanoclusters enhances the $\mathrm{NO}_{2}$ gas sensitivity up to the detection of sub-ppm level of great interest for selective environmental $\mathrm{NO}_{2}$ air monitoring. The effects of tailored $\mathrm{Au}$ loading onto the CNT surface on $\mathrm{NO}_{2}$ gas sensitivity depend on nanoclusters density and sensor working temperature. An excellent short-term repeatability of the response to the selected targeted gases has been also measured for the $\mathrm{Au}$ modified CNT-based sensors. A continuous gas monitoring at ppb level of the $\mathrm{NO}_{2}$ gas has been effectively performed with CNT chemiresistors modified with a 0.3 at. \% Au loading, at sensor temperature of $150^{\circ} \mathrm{C}$. The p-type character of the Au-modified CNT sensors has been also confirmed. Moreover, no cross-sensitivity has been revealed for the investigated gases, since all CNT-based devices show a higher selectivity for $\mathrm{NO}_{2}$ gas.

Finally, the proposed electrochemical functionalization process of the CNTs seems to be easily applicable for lowcost mass production of modified CNT-based gas sensors.

Future work on different metal surface-modifications of the carbon nanotube networked films is planned for specific gas detection in sensor arrays concerning environmental monitoring applications.

Acknowledgements. The authors thank Gennaro Cassano for technical assistance during sensing experiments in ENEA. Authors acknowledge the Ministry of University and Scientific Research (MIUR) of Italy, PON program 2007-2013 for financial support. Finally, the authors are indebted to the COST Action TD1105 EuNetAir for the international networking activities in the field of the sensor materials for air pollution monitoring.

Edited by: A. Lloyd Spetz

Reviewed by: two anonymous referees

\section{References}

Bondavalli, P., Legagneux, P., and Pribat, D.: Carbon nanotubes basedtransistors as gas sensors: State of the art and critical review, Sensor Actuat. B-Chem., 140, 304-18, 2009.

Brunet, J., Dubois, M., Pauly, A., Spinelle, L., Ndiaye, A., Guérin, K., Varenne, C., Lauron, B.: An innovative gas sensor system designed from a sensitive organic semiconductor downstream a nanocarbonaceous chemical filter for the selective detection of $\mathrm{NO}_{2}$ in an environmental context Part I: Development of a nanocarbon filter for the removal of ozone, Sensors Actuat. BChem., 173, 659-667, 2012.

Chang, H., Lee, J. D., Lee, S. M., and Lee, Y. H.: Adsorption of $\mathrm{NH}_{3}$ and $\mathrm{NO}_{2}$ molecules on carbon nanotubes, Appl. Phys. Lett. 79, 3863-3865, 2001.

Charlier, J.-C.: Defects in Carbon Nanotubes, Acc. Chem. Res., 35, 1063-1069, 2002.

Cioffi, N., Torsi, L., Sabbatini, L., Zambonin, P. G., and BleveZacheo, T.: Electrosynthesis and characterisation of nanostructured palladium-polypyrrole composites, J. Electroanal. Chem., 488, 42-47, 2000.

Cioffi, N., Torsi, L., Losito, I., Sabbatini, L., Zambonin, P. G., and Bleve-Zacheo, T.: Nanostructured palladium-polypyrrole composites electrosynthesised from organic solvents, Electrochim. Acta, 46, 4205-4211, 2001.

Colindres, S. C., Aguir, K., Sodi, F. C., Vargas, L. V., Salazar, J. A. M., and Febles, V. G.: Ozone Sensing Based on Palladium Decorated Carbon Nanotubes, Sensors, 14, 6806-6818, 2014.

Collins, P. G., Bradley, K., Ishigami, M., and Zettl, A.: Extreme Oxygen Sensitivity of Electronic Properties of Carbon Nanotubes, Science, 287, 1801-1804, 2000.

Doroodmand, M. M., Nasresfahani, S., and Sheikhi, M. H.: Fabrication of ozone gas sensor based on $\mathrm{FeOOH} /$ single walled carbon nanotube-modified field effect transistor, Int. J. Environ. Anal. Chem., 93, 946-958, 2013.

Espinosa, E. H., Ionescu, R., Bittencourt, C., Felten, A., Erni, R., Van Tenderloo, G., Pireaux, J.-J., and Llobet, E.: Metal-decorated multi-wall carbon nanotubes for low temperature gas sensing, Thin Solid Films, 515, 8322-8327, 2007.

Fan, Y., Goldsmith, B. R. and Collins, P. J.: Identifying and counting point defects in carbon nanotubes, Nature Mater., 4, 906-911, 2005.

Feldheim, D. L. and Foss, C. A. (Eds.): Metal Nanoparticles: Synthesis, Characterization and Applications, New York: Marcel Dekke, 2002.

Hvolbæk, B., Janssens, T. V. W., Clausen, B. S., Falsig, H., Christensen, C. H., and Nørskov, J. K.: Catalytic activity of $\mathrm{Au}$ nanoparticles, NanoToday, 2, 14-18, 2007.

Ieva, E., Buchholt, K., Colaianni, L., Cioffi, N., Sabbatini, L., Capitani, G. C., LloydSpetz, A., Kall, P. O., and Torsi, L.: Au Nanoparticles as Gate Material for NOx Field Effect Capacitive Sensors, Sens. Lett., 6, 1-8, 2008.

Kauffman, D. R., Sorescu, D. C., Schofield, D. P., Allen, B. L., Jordan, K. D., and Star, A.: Understanding the Sensor Response of Metal-Decorated Carbon Nanotubes, Nano Lett. 10, 958-963, 2010.

Khalap, V. R., Sheps, T.ì Kane, A. A., and Collins, P. G.: Hydrogen Sensing and Sensitivity of Palladium-Decorated Single- Walled Carbon Nanotubes with Defects, Nano Lett., 10, 896-901, 2010. 
Kim, S. J., Park, Y. J., Ra, E. J., Kim, K. K., An, K. H., Lee, Y. H., Choi, J. Y., Park, J. C., Doo, S. K., Park, M. H., and Yang, C. W.: Defect-induced loading of Pt nanoparticles on carbon nanotubes, Appl. Phys. Lett., 90, 023114-023116, 2007.

Kong, J.: Nanotube molecular wires as chemical sensors, Science, 287, 622-625, 2000.

Kong, J., Chapline, M. G., and Dai, H.: Functionalized Carbon Nanotubes for Molecular Hydrogen Sensors, Adv. Mater., 13, 13841386, 2001.

Leghrib, R., Felten, A., Demoisson, F., Reniers, F., Pireaux, J.-J., and Llobet, E.: Room-tempeature, selective detection of benzene at trace levels using plasma-treated metal-decorated multiwalled carbon nanotubes, Carbon 48, 3477-3484, 2010.

Lu, Y., Li, J., Han, J., Ng, H.-T., Binder, C., Partridge C., and Meyyappan, M.: Room temperature methane detection using palladium loaded single-walled carbon nanotube sensors, Chem. Phys. Lett., 391, 344-348, 2004.

Lv, J.-A., Cui, J.-H., Li , X-.N., Song, X.-Z., Wang, J.-G., and Dong, M.: The point-defect of carbon nanotubes anchoring Au nanoparticles, Physica E, 42, 1746-1750, 2010.

Martel, R., Schmidt, T., Shea, H. R., and Avouris, P.: Single- and multi-wall carbon nanotube field-effect transistors, Appl. Phys. Lett., 73, 2447-2449, 1998.

Metal Nanoparticles: Synthesis, Characterization and Applications, edited by: Foss, C. J. and Feldheim, D., Marcel Dekker, New York, 2002.

Moulder, J. F., Stickle, W. F., Sobol, P. E., and Bomben, K. D.: Handbook of X-Ray Photoelectron Spectroscopy, Perkin-Elmer Corporation, Eden Praire, Minnesota, 1992.

Mubeen, S., Lim, J.-H., Srirangarajan, A., Mulchandani, A., Deshusses, M. A., and Myung N. V.: Gas sensing mechanism of gold nanoparticles decorated single-walled carbon nanotubes, Electroanalysis, 23, 2687-92, 2011.

Pannopard, P., Khongpracha, P., Probst, M., and Limtrakul, J.: Gas sensing properties of platinum derivatives of singlewalled carbon nanotubes: a DFT analysis, J. Mol. Graph. Modelling, 28, 62-69, 2009.

Peng, N., Zhang, Q., Chow, C. L., Tan, O. K., and Marzari, N.: Sensing mechanisms for carbon nanotube based $\mathrm{NH}_{3}$ gas detection, Nano. Lett., 9, 1626-1630, 2009.

Peng, S. and Cho, K.: Ab Initio Study of Doped Carbon Nanotube Sensors, Nano Lett., 3, 513-517, 2003.

Penza, M., Cassano, G., Rossi, R., Alvisi, M., Rizzo, A., Signore, M. A., Dikonimos, Th., Serra, E., and Giorgi, R.: Enhancement of sensitivity in gas chemiresistors based on carbon nanotube surface functionalized with noble metal (Au, Pt) nanoclusters, Appl. Phys. Lett., 90, 173123-173125, 2007.

Penza, M., Rossi, R., Alvisi, M., Cassano, G., Signore, M. A., Serra, E., and Giorgi, R.: Surface Modification of Carbon Nanotube Networked Films with Au Nanoclusters for Enhanced NO2 Gas Sensing Applications, J. Sensors, 2008, 107057, doi:10.1155/2008/107057, 2008.
Penza, M., Rossi, R., Alvisi, M., and Serra, E., Metal-modified and vertically aligned carbon nanotube sensors array for landfill gas monitoring applications, Nanotechnology, 21, 105501-1055014, 2010.

Penza, M., Rossi, R., Alvisi, M., Suriano, D., and Serra, E.: Ptmodified carbon nanotube networked layers for enhanced gas microsensors, Thin Solid Films, 520, 959-65, 2011.

Penza, M., Martin, P., and Yeow, J.: Carbon Nanotube Gas Sensors, in: Gas Sensing Fundamentals, edited by: Kohl, C.-D. and Wagner, T., Springer Series on Chemical Sensors and Biosensors, 15, 109-114, 2014.

Radnik, J., Mohr, C., and Claus, P.: The origin of binding energy shifts of core levels of supported gold nanoparticles and dependence of pretreatment and material synthesis, Phys. Chem. Chem. Phys., 5, 172-177, 2003.

Reetz, M. T. and Helbig, W.: Size-Selective Synthesis of Nanostructured Transition Metal Clusters, J. Am. Chem. Soc., 116, 74017402, 1994.

Reetz, M. T., Helbig, W., Quaiser S.A., Stimming U., Breuer, N., and Vogel R.: Visualization of surfactants on nanostructured palladium clusters by a combination of STM and high-resolution TEM, Science, 267, 367-369, doi:10.1126/science.267.5196.367, 1995.

Robinson, J. A., Snow, E. S., Badescu, S. C, Reinecke, T. L., and Perkins, F. K.: Role of Defects in Single-Walled Carbon Nanotube Chemical Sensors, Nano Lett., 6, 1747-1751, 2006.

Salavagione, H. J., Dìez-Pascual, A. M., Lazaro, E., Vera, S., and Gomez-Fatou, M. A.: Chemical sensors based on polymer composites with carbon nanotubes and graphene: the role of the polymer, J. Mater. Chem. A, 2, 14289-14328, doi:10.1039/C4TA02159B, 2014.

Scarselli, M., Camilli, L., Castrucci, P., Nanni, F., Del Gobbo, S., Gautron, E., Lefrant, S., and De Crescenzi, M.: In situ formation of noble metal nanoparticles on multiwalled carbon nanotubes and its implication in metal-nanotube interactions, Carbon, 50, 875-84, 2012.

Star, A., Joshi, V., Skarupo, S., Thomas, D., and Gabriel, J.-C. P.: Gas sensor array based on metal-decorated carbon nanotubes, J. Phys. Chem. B, 110, 21014-21020, 2006.

Zhang, T., Mubeen, S., Myung, N. V., and Deshusses, M. A.: Recent progress in carbon nanotube-based gas sensors, Nanotechnology, 19, 332001-332014, 2008.

Zhang, X., Dai, Z., Chen, Q., and Tang, J.: A DFT study of $\mathrm{SO}_{2}$ and $\mathrm{H}_{2} \mathrm{~S}$ gas adsorption on Au-doped single-walled carbon nanotubes, Phys. Scr., 89, 065803-065809, 2014.

Zhang, Y., Cui, S., Chang, J., Ocola, L. E., and Chen, J.: Highly sensitive room temperature carbon monoxide detection using $\mathrm{SnO}_{2}$ nanoparticle-decorated semiconducting single-walled carbon nanotubes, Nanotechnology, 24, 025503-025512, 2013.

Zhao, J., Buldum, A., Han, J., and Lu, J. P.: Gas molecule adsorption in carbon nanotubes and nanotube bundles, Nanotechnology, 13, 195-200, 2002. 